\title{
VIÊC SỬ DỤNG CÁC LOẠI LỖ TRONG LIÊN KẾT BU LÔNG CƯỜNG Độ CAÖ TRONG XÂY DỰNG CÂU THÉP THEO 22TCN 272-05
}

\author{
ĐỖ CAO PHAN ${ }^{1}$, NGUYỄN ĐĂNG ĐIỀ ${ }^{2}$ \\ ${ }^{1}$ Truòng đại học Công nghiệp thành phố Hồ Chi Minh, \\ ${ }^{2}$ Phân hiệu truờng Đại học Giao thông vận tải tại thành phố Hồ Chí Minh; \\ docaophan@iuh.edu.vn,dangdiem510@gmail.com
}

Tóm tắt.Theo tiêu chuẩn thiết kế cầu 22TCN 272-05, liên kết bu lông cường độ cao chỉ được phép sử dụng lỗ tiêu chuẩn. Tuy nhiên, trong thực tế thi công, nhiều trường hợp đơn vị thi công khoan lỗ sai quy cách và không được phép sử dụng theo tiêu chuẩn. Bài báo này đề xuất sử dụng các loại lỗ trong liên kết bu lông cường độ cao dựa trên phân tích ứng suất cục bộ tại mép lỗ bằng phương pháp phần tử hữu hạn.

Từ khóa.22TCN272-05, bu lông cường độ cao, lỗ bu lông, thép.

\section{USING THE HOLES IN THE HIGH-STRENGTH BOLT SPLICE IN STEEL BRIDGE CONSTRUCTION IN 22TCN 272-05}

\begin{abstract}
According to 22TCN 272-05, high-strength bolts splice are allowed only to use the standard hole. However, in reality, many contractors drilled a incorrect holes and aren't allowed to use by the standard. This paper proposes to use the holes in the high-strength bolts splice, based on a local stress analysis at the edge of the hole by the Finite Element Method.
\end{abstract}

Keywords. 22 TCN 272-05, bolt - hole, high-strength bolt, steel.

\section{GIỚI THIỆU}

Đối với kết cấu thép hiện đại, liên kết bu lông được sử dụng rộng rãi. Liên kết bu lông có độ tin cậy cao, dễ thi công, thay thế và sửa chữa.Tuy nhiên để liên kết phải khoan lỗ trên bề mặt thép cơ bản, những khu vực này gián đoạn về mặt hình học nên xuất hiện ứng suất tập trung tại mép lỗ. Theo [1], khi liên kết kết cấu thép bằng liên kết bu lông thì có các dạng lỗ khoan sau đây:

Bảng 1. Các loại lỗ bu lông theo 22TCN 272-05[1].

\begin{tabular}{|c|c|c|c|c|}
\hline \multirow[b]{2}{*}{$\begin{array}{l}\text { Đường kính bu } \\
\text { lông }(\mathrm{mm})\end{array}$} & Tiêu chuẩn & Quá cỡ & Rãnh ngắn & Rãnh dài \\
\hline & $\begin{array}{l}\text { Đường kính } \\
\text { (mm) }\end{array}$ & $\begin{array}{l}\text { Đường kính } \\
\text { (mm) }\end{array}$ & $\begin{array}{l}\text { Rộng x Dài } \\
\text { (mm x mm) }\end{array}$ & $\begin{array}{l}\text { Rộng x Dài } \\
\text { (mm x mm) }\end{array}$ \\
\hline 16 & 18 & 20 & $18 \times 22$ & $18 \times 40$ \\
\hline 20 & 22 & 24 & $22 \times 26$ & $22 \times 50$ \\
\hline 22 & 24 & 28 & $24 \times 30$ & $24 \times 55$ \\
\hline 24 & 26 & 30 & $26 \times 33$ & $26 \times 60$ \\
\hline 27 & 30 & 35 & $30 \times 37$ & $30 \times 67$ \\
\hline 30 & 33 & 38 & $33 \times 40$ & $33 \times 75$ \\
\hline 36 & 39 & 44 & $39 \times 46$ & $39 \times 90$ \\
\hline
\end{tabular}

Theo [1], đối với liên kết bu lông thường, các lỗ trên có thể được sử dụng trong bất cứ lớp nào hoặc tất cả các lớp của các liên kết ma sát trượt hoặc ép tựa. Với các rãnh có thể được sử dụng mà không cần chú ý đến phương của tải trọng trong các liên kết trượt tới hạn, nhưng chiều dài phải trực giao với phương của tải trọng trong các liên kết kiểu ép tựa.Còn với liên kết bu lông cường độ cao thì phải sử dụng các lỗ tiêu chuẩn.Khi tính toán sức kháng của liên kết bu lông cường độ cao ở trạng thái giới hạn cường độ bao gồm: sức kháng cắt tính toán của bu lông, sức kháng cắt khối và sức kháng ép mặt tính toán của thép cơ bản. Sức kháng ép mặt tính toán của thép cơ bản được xác định đối với các loại lỗ chuẩn, lỗ quá cỡ, lỗ rãnh ngắn chịu tác dụng lực theo mọi phương và lỗ rãnh dài song song với phương tác dụng của lực là 
giống nhau. Khi xem xét liên kết trong toàn bộ cấu kiện thì 22TCN 272-05 có xét đến hiện tượng tập trung ứng suất thông qua hệ số chiết giảm $U$, hệ số $U$ phụ thuộc vào hình thức liên kết và cấu tạo của liên kết mà chưa đề cập đến loại lỗ của liên kết. Trong thực tế, nhiều trường hợp các đơn vị thi công khoan lỗ để liên kết bu lông cường độ cao không phải dạng lỗ tiêu chuẩn và vẫn sử dụng trong kết cấu.Theo hiểu biết của tác giả, ở Việt Nam, hiện chưa có nghiên cứu nào về việc sử dụng các loại lỗ trong liên kết bu lông cường độ cao. Trên thế giới có nghiên cứu về ứng suất cục bộ xung quanh các lỗ bu lông của mối nối đường ray[2] hay hay sự ảnh hưởng qua lại giữa hai lỗ trên chi tiết [3].Bài báo dựa trên phân tích ứng suất cục bộ tại mép lỗ bu lông đối với các loại lỗ khác nhau bằng phương pháp phần tử hữu hạn thông qua phần mềm Midas FEA, từ đó đề xuất sử dụng các loại lỗ trong liên kết bu lông cường độ cao.

\section{CƠ SỞ LÝ THUYẾT}

Cơ sở lý thuyết tính toán là phương pháp phần tử hữu hạn, áp dụng cho bài toán ứng suất phẳng, sử dụng phần tử tam giác bậc hai.Hàm chuyển vị là đa thức bậc hai đầy đủ, với mô hình này ứng suất trong phần tử sẽ thay đổi tuyến tính phản ánh tốt trạng thái ứng suất của phần tử, nó cũng thích hợp với dạng biên cong của phần tử.

Phần tử tam giác bậc hai gồm 6 nút: 3 nút ở đỉnh của tam giác và 3 nút ở trung điểm của các cạnh tam giác, hàm chuyển vị được xấp xỉ [4]:

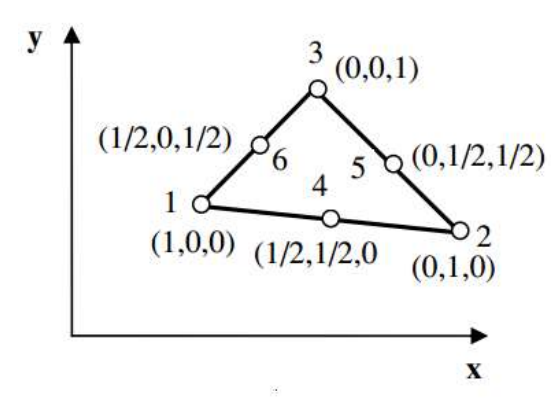

Hinh 1. Phần tử tam giác trong hệ tọa độ tự nhiên.

$$
\left\{\begin{array}{l}
u(x, y)=a_{1}+a_{2} x+a_{3} y+a_{4} x y+a_{5} x^{2}+a_{6} y^{2} \\
v(x, y)=a_{7}+a_{8} x+a_{9} y+a_{10} x y+a_{11} x^{2}+a_{12} y^{2}
\end{array}\right.
$$

Viết dưới dạng ma trận: $\{\mathrm{f}\}=\left\{\begin{array}{l}\mathrm{u}(\mathrm{x}, \mathrm{y}) \\ \mathrm{v}(\mathrm{x}, \mathrm{y})\end{array}\right\}=[N]\{\delta\}_{e}$

Với: $\quad-[\mathrm{N}]=\left[\begin{array}{cccccccccccc}\mathrm{N}_{1} & \mathrm{~N}_{2} & \mathrm{~N}_{3} & \mathrm{~N}_{4} & \mathrm{~N}_{5} & \mathrm{~N}_{6} & 0 & 0 & 0 & 0 & 0 & 0 \\ 0 & 0 & 0 & 0 & 0 & 0 & \mathrm{~N}_{1} & \mathrm{~N}_{2} & \mathrm{~N}_{3} & \mathrm{~N}_{4} & \mathrm{~N}_{5} & \mathrm{~N}_{6}\end{array}\right]$ : Ma trận hàm dạng.

- $\{\delta\}_{e}$ : Véc tơ chuyển vị nút phần tử

Ma trận độ cứng của phần tử được xác định: $[K]=\iint[B]^{T}[D][B] d v=\iint[B]^{T}[D][B] t d x d y$

Với: $\quad-[D]=\frac{E}{1-v^{2}}\left[\begin{array}{ccc}1 & v & 0 \\ v & 1 & 0 \\ 0 & 0 & \frac{1-v}{2}\end{array}\right]$ : Ma trận các hệ số đàn hồi với bài toán ứng suất phẳng.

- $[B]=[\nabla][N]=\left[\begin{array}{cc}\frac{\partial}{\partial x} & 0 \\ 0 & \frac{\partial}{\partial y} \\ \frac{\partial}{\partial y} & \frac{\partial}{\partial x}\end{array}\right][N]:$ Ma trận biến dạng.

- t: Chiều dày của tầm 
Véc tơ tải trọng nút được xác định: $\{P\}=\int[N]^{T}\{p\} d V$

Với: $\quad-\{p\}$ : Véc tơ tải tác dụng lên phần tử

Úng suất của phần tử được xác định:

$$
\left\{\begin{array}{c}
\sigma_{x} \\
\sigma_{y} \\
\tau_{x y}
\end{array}\right\}=[D] \times[B] \times\{\delta\}_{e}=\frac{E}{1-v^{2}}\left[\begin{array}{ccc}
1 & v & 0 \\
v & 1 & 0 \\
0 & 0 & \frac{1-v}{2}
\end{array}\right] \times[B] \times\{\delta\}_{e}
$$

\section{VÍ DỤ ÁP DỤNG}

\subsection{Mô hình hóa}

Như đã trình bày ở phần 2 , trong mục này sẽ tính toán ứng suất cục bộ tại mép lỗ bu lông của tấm thép dưới tác dụng của tải trọng. Phương pháp phần tử hữu hạn được sử dụng thông qua phần mềm Midas FEA.

Trong phạm vi bài báo này tác giả tính toán cho tấm có kích thước $100 \mathrm{mmx} 100 \mathrm{~mm}$ và chiều dày là $12 \mathrm{~mm}$, vật liệu thép được đưa vào tính toán có mô đun đàn hồi $\mathrm{E}=2 \times 10^{5} \mathrm{MPa}$, hệ số Poisson $v=0,25$. Do tác dụng của mọi loại đường kính bu lông lên mép lỗ là như nhau nên tác giả sử dụng bu lông cường độ cao có đường kính $\mathrm{d}=22 \mathrm{~mm}$ trong liên kết tính toán. Theo[1] quy định tương quan về đường kính lỗ bu lông và đường kính thân bu lông, do đó phần tiếp xúc giữa thân bu lông và tấm kim loại được xem như một hình chữ nhật có kích thước là chiều dày tấm nhân với phần tiếp xúc. Trong phạm vi bài báo tác giả chưa xét đến ảnh hưởng ở mức độ khác nhau của các lọa đường kính lỗ, đây sẽ là hướng nghiên cứu tiếp theo của nhóm tác giả. Tấm được chia thành các phần tử tam giác, các phần tử tam giác được sử dụng là phần tử bậc hai với 6 nút tại đỉnh và trung điểm các cạnh tam giác. Chiều dày của tấm $12 \mathrm{~mm}$ được xem là mỏng so với kích thước của tấm, tải trọng tác dụng nằm trong mặt phẳng tấm nên đây là bài toán ứng suất phẳng. Bài toán đối xứng theo hai trục, để đơn giản và giảm khối lượng tính toán bài toán được mô hình hóa là một phần tư lỗ bu lông. Điều kiện biên được đưa vào tại các mép lỗ là liên kết hạn chế chuyển vị đường, các mặt còn lại là biên tự do. Theo $22 \mathrm{TCN} 272-05$ khi sử dụng bu lông có đường kính $\mathrm{d}=22 \mathrm{~mm}$ thì có các dạng lỗ khoan là:

- Lỗ tròn tiêu chuẩn có đường kính $\mathrm{d}_{1}=24 \mathrm{~mm}$ (Lỗ $\left.\mathrm{TC}\right)$.

- Lỗ tròn quá cỡ có đường kính $\mathrm{d}_{2}=28 \mathrm{~mm}$ (Lỗ $\left.\mathrm{QC}\right)$.

- Lỗ elip rãnh ngắn rộng x dài $=24$ x 30 mm, khoan theo phương ngang (Lỗ E2430n).

- Lỗ elip rãnh ngắn rộng x dài $=24$ x 30 mm, khoan theo phương dọc (Lỗ E2430d).

- Lỗ elip rãnh dài rộng x dài $=24$ x 55 mm, khoan theo phương ngang (Lỗ E2455n).

- Lỗ elip rãnh dài rộng x dài = 24 x 55 mm, khoan theo phương dọc (Lỗ E2455d).

Trong thực tế thi công, đơn vị thi công ngoài khoan lỗ tiêu chuẩn còn khoan các lỗ sai quy cách. Các dạng lỗ sai quy cách có thể là các lỗ tròn lệch tâm, lỗ tròn đúng tâm có đường kính lớn hay nhỏ hơn lỗ tiêu chuẩn, lỗ không tròn,... và để tổng quát cho các kiểu lỗ sai quy cách, tiêu chuẩn 22TCN 272-05 đã quy định các loại lỗ sai quy cách đó là lỗ quá cỡ, lỗ elip rãnh dài và lỗ elip rãnh ngắn.Trong bài báo này, tác giả đã so sánh ứng suất cục bộ của lỗ sai quy cách so với lỗ tiêu chuẩn, qua đó đề xuất sử dụng các loại lỗ sai quy cách trong liên kết bu lông cường độ cao.

Cấu tạo của các tấm thép dùng để phân tích cục bộ như hình 2 . 

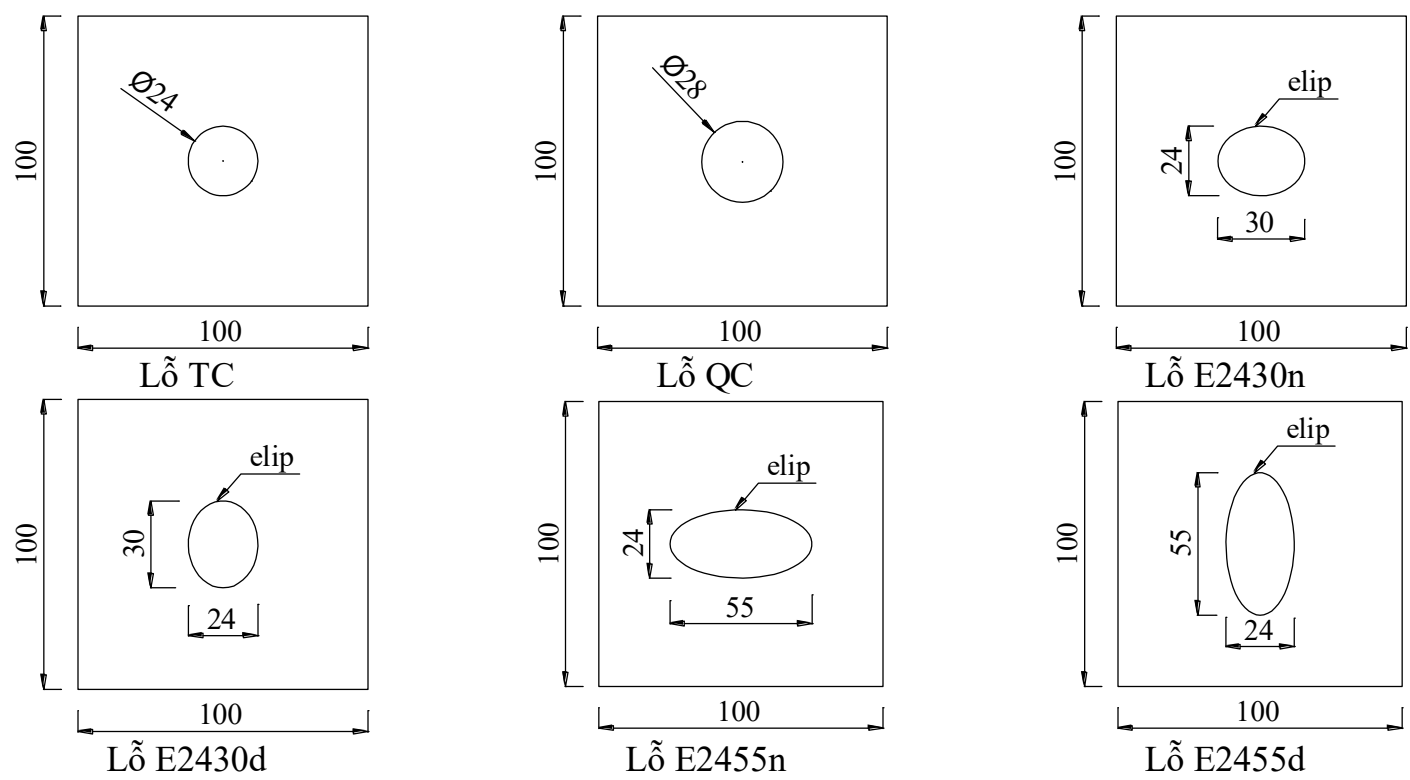

Hinh 2. Cấu tạo các các lỗ khoan dùng cho bu lông đường kính 22mm theo 22TCN 272-05.

Do các tấm là đối xứng theo hai phương nên khi phân tích ta chỉ phân tích cho $\frac{1}{4}$ tấm, tấm chịu tải trọng phân bố đều theo phương y trên mép theo trục $x$ của tấm, tải trọng có độ lớn bằng $20 \mathrm{kN} / \mathrm{m}$ (tấm chịu ứng suất kéo)

\subsection{Kết quả}

Sử dụng phương pháp đã trình bày ở mục 2 , ta thu được kết quả như sau:

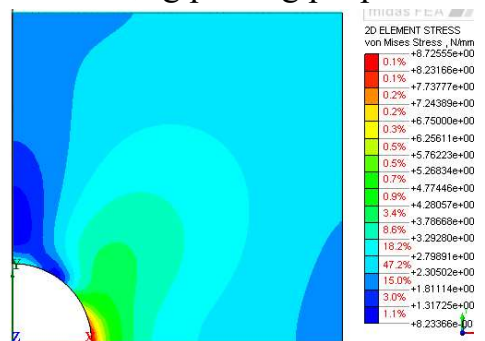

Lỗ TC

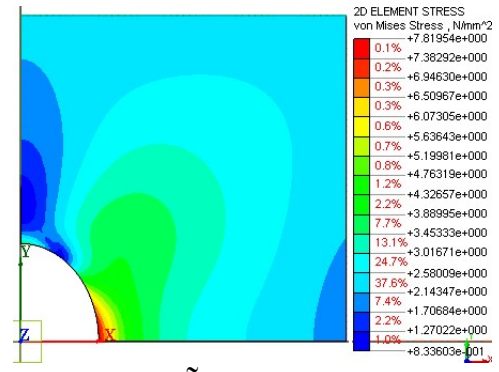

Lỗ E2430d

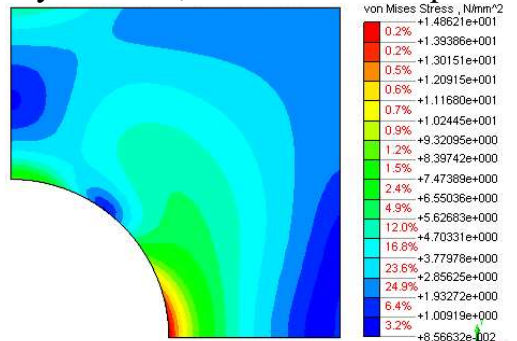

Lỗ QC

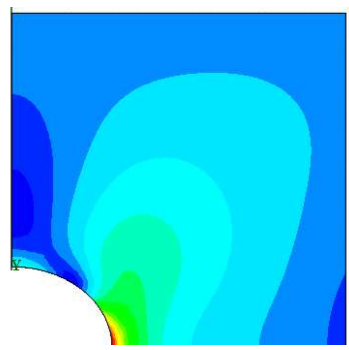

Lỗ E2455n

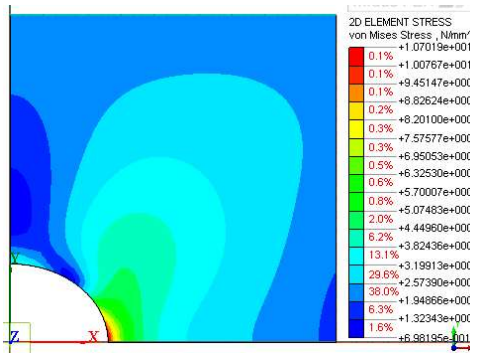

Lỗ E2430n
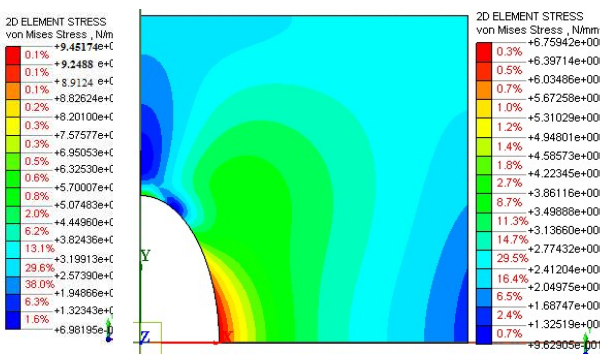

Lỗ E2455d

Hình 3: Kết quả phân tích ứng suất cục bộ.

Hình 3 là phân bố ứng suất cục bộ tại trên bản thép dùng để liên kết bu lông cường độ cao, ta thấy ứng suất tập trung tại mép lỗ lớn nhất theo phương $\mathrm{x}$ và giảm rất nhanh khi điểm tính ứng suất càng xa lỗ khoan. 


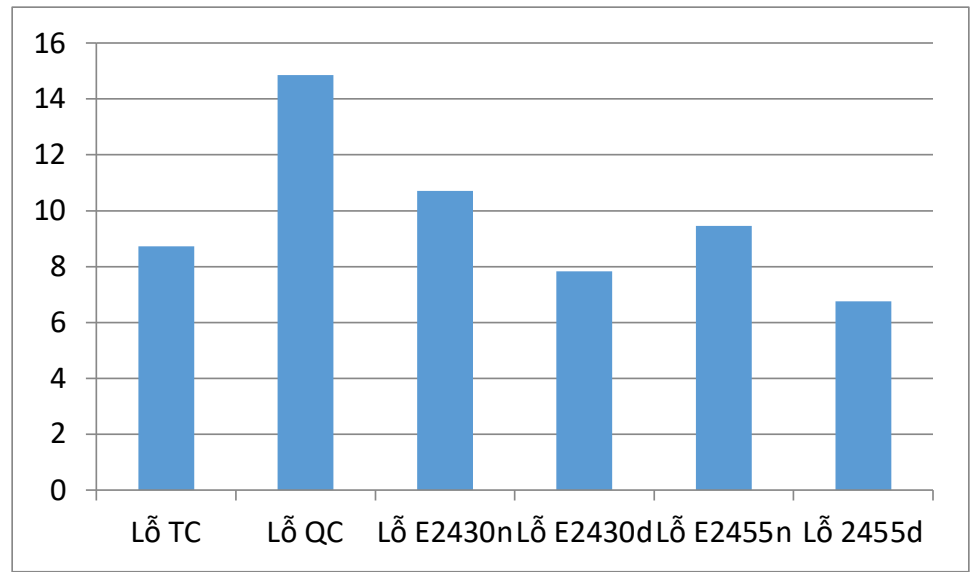

Hinh 4. So sánh ứng suất cục bộ lớn nhất tại mép lỗ.

Hình 4 là so sánh ứng suất lớn nhất trên mép lỗ của các loại lỗ, trong đó lỗ QC, lỗ E2430n và lỗ E2455n có ứng suất tập trung lớn nhất và dễ bị xé rách trong các liên kết chịu lực theo phương $\mathrm{x}$.

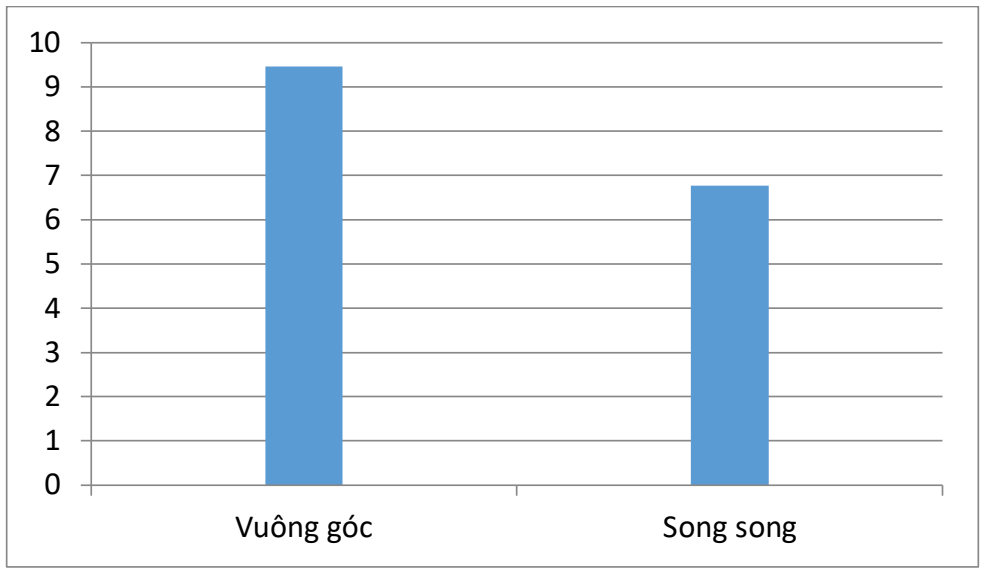

Hình 5. Ứng suất cục bộ của lỗ rãnh ngắn $24 \times 30 \mathrm{~mm}$.

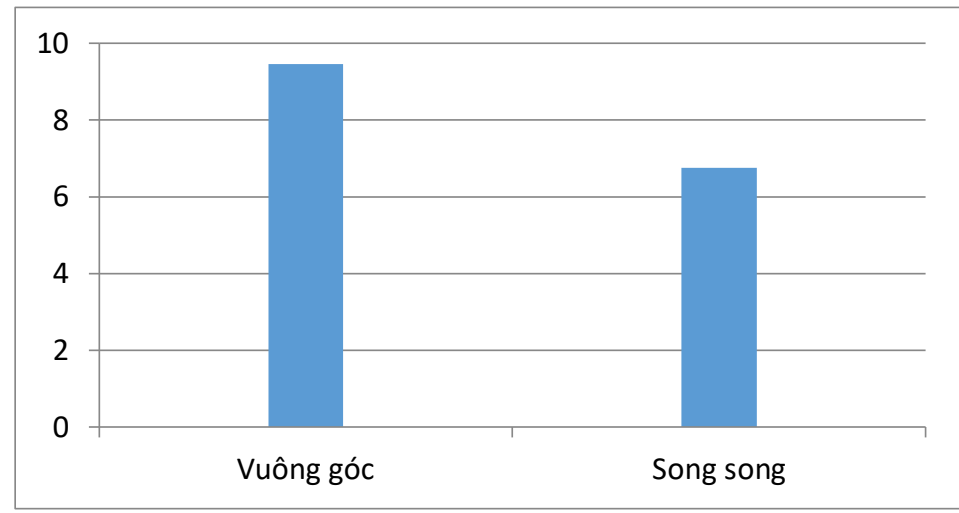

Hình 6. Ứng suất cục bộ của lỗ rãnh dài 24x55mm.

Hình 5 và hình 6 là so sánh ứng suất cục bộ lớn nhất của hai dạng lỗ elip trong trường hợp cạnh dài của elip nằm song song và vuông góc với phương tác dụng của lực.Với trường hợp cạnh dài elips nằm 
vuông góc với phương tác dụng của lực thì cho ứng suất cục bộ lớn hơn.Qua đó ta nên hạn chế sử dụng lỗ elip có cạnh dài vuông góc với phương tác dụng của lực để tránh trường hợp lỗ bị phá hoại.

Tóm lại, ta nhận thấy rằng ứng suất tập trung ở mép lỗ theo phương vuông góc với tải trọng tác dụng, đối với các loại lỗ khác nhau thì giá trị và vùng phân bố ứng suất có khác nhau, ứng suất tập trung lớn nhất đối với lỗ quá cỡ. Đối với cùng loại lỗ e-líp khi cạnh dài vuông góc với phương tác dụng của lực thì ứng suất cục bộ lớn hơn so với trường hợp cạnh dài dọc theo phương tác dụng của lực.

Bảng 2. Kết quả phân tích cục bộ đối với các loại lỗ khác nhau.

\begin{tabular}{|l|c|c|c|}
\hline \multicolumn{1}{|c|}{ Loại lỗ khoan } & Úng suất lớn nhất $\left(\mathrm{N} / \mathrm{mm}^{2}\right)$ & Tỷ lệ \% ứng suất lớn nhất & So sánh \\
\hline Lô̂̃ TC & $+8.7255 \mathrm{e}+0$ & $0.1 \%$ & 1 \\
\hline Lỗ QC & $+1.4862 \mathrm{e}+1$ & $0.2 \%$ & 1.703 \\
\hline Lỗ E2430n & $+1.0702 \mathrm{e}+1$ & $0.1 \%$ & 0.896 \\
\hline Lỗ E2430d & $+7.8195 \mathrm{e}+0$ & $0.1 \%$ & 1.083 \\
\hline Lỗ E2455n & $+9.4517 \mathrm{e}+0$ & $0.1 \%$ & 0.775 \\
\hline Lỗ E2455d & $+6.7594 \mathrm{e}+0$ & $0.3 \%$ & \\
\hline
\end{tabular}

Từ bảng 2, ta thấy lỗ quá cỡ có ứng suất tập trung lớn nhất trong các loại lỗ (gấp 1.703 lần so với ứng suất của lỗ tiêu chuẩn và giá trị ứng suất lớn nhất trong biểu đồ ứng suất chiếm $0.2 \%$ ), vì vậy nên sử dụng lỗ quá cỡ cho các lớp liên kết chịu ma sát, khi đó các bản ép vào nhau nhờ lực xiết trong các bu lông, lực ép giữa các bản đủ lớn không cho các bản trượt.Không dùng lỗ quá cỡ cho liên kết làm việc ép mặt khi đó thân bu lông ép mặt lên thép cơ bản tạo ra ứng suất cục bộ lớn tại mép lỗ.

Hệ số chiết giảm $U$ do hiện tượng tập trung ứng suất khi tính toán cấu kiện chịu kéo cần được lấy giá trị nhỏ hơn so với liên kết bu lông sử dụng lỗ chuẩn.

\section{KẾT LUẬN}

Từ kết quả phân tích ở trên, tác giả nhận thấy có thể sử dụng lỗ chuẩn, lỗ elip cạnh ngắn và cạnh dài cho các liên kết bu lông cường độ cao chịu ma sát hoặc ép mặt. Tuy nhiên khi sử dụng lỗ elip cạnh ngắn và dài cho liên kết chịu ép mặt thì phải chú ý đặt cạnh dài song song với phương tác dụng của lực. Đối với lỗ quá cỡ thì chỉ được dùng trong liên kết bu lông cường độ cao chịu ma sát.

\section{TÀI LIỆU THAM KHẢO}

[1] Bộ giao thông vận tải, Tiêu chuẩn thiết kế cầu 22TCN 272-05, Nhà xuất bản Giao thông vận tải, 2005

[2] Hiroo KATAOKA, Evaluation of stresses around fish bolt holes of jointed rails, $7^{\text {th }}$ International Heavy Haul Conference, 2001.

[3] W.Z.Zhuang, Prediction of rack growth from bolt holes in a disc, international Joural of Fatigue, 2000.

[4] Daryl L.Logan, A first course in the finite element method, fifth edition.

[5] Midas Information Technology Co.,Ltd., Midas FEA - Analysis and Algorithm, Korea.

Ngày nhận bài: 29/09/2017

Ngày chấp nhận đăng: 28/12/2017 